\title{
Eine neue Discoptila aus anatolischen Höhlen (Orthoptera, Gryllidae)
}

\author{
von \\ Alexi POPOV* \\ SUMMARY
A new Discoptila from anatolian caves
(Orthoptera, Gryllidae)

\begin{abstract}
A new troglophilous cave-cricket, Discoptila beroni n.sp. is described and figured. It is common in the caves Maara near Mersin and Damlatas near Alanya in South Anatolia. The characteristic features of the males, as well as of the females, by which the new species is distinguished from the other eight species of the genus, all distributed in the Mediterranean, are pointed out. $D$. beroni $\mathrm{n}$.sp. has already been reported erroneously from the cave Damlatas as D. fragosoi (Bol.) and as D. brevis B.-Bien.
\end{abstract}

Im Jahre 1972 unternahmen meine Kollegen und Freunde, die Herren Vl.Beschkov, T. Mitschev und P. Beron, eine zweimonatige Reise nach Vorderasien, während der sie Fauna in einigen türkischen Höhlen sammelten. In zwei von ihnen fanden sie eine Höhlengrille aus der Gattung Discoptila. Für die liebenswürdig mir zur Verfügung gestellten Exemplare dieser Art danke ich ihnen noch einmal. Es erwies sich, dass diese Discoptila mit keiner der bis jetzt bekannten 8 Arten dieser Gattung, in Südeuropa und Nordafrika verbreitet, identisch ist. Die neue Art wird im folgenden beschrieben.

\section{Discoptila beroni n.sp.}

Discoptila fragosoi Weidner 1964: 17-19 (nec Bolivar 1885)

Discoptila brevis Harz 1969: 707 (nec Bey-Bienko 1964)

Holotypus o: Türkei, Südanatolien, Höhle Maara, beim Dorf Karatepe, Kaza (Bezirk) Gülnar, Vil. (Prov.) Icel, 15. Dezember 1972, leg. P. Beron und V. Beschkov; Allotypus $\$$; gleicher Fundort, Datum und Sammler; Paratypen 6 ơ und 5\%?: gleicher Fundort, Datum und Sammler; Paratypen 2 ơ und 2\%o: Türkei, Südanatolien, Höhle Damlatas (= Damlatasch), Umgebung von Alanya, Vil. Antalya, 16.Dezember 1972, leg. P. Beron, V. Beschkov und T. Mitschev.

Weiteres Material: 1 o ad., 1 o juv. und 3 o juv. aus der Höhle Maara und $1 \delta^{\star}$ juv. aus der Höhle Damlatas werden nicht als Paratypen bezeichnet. Das erwachsene

\footnotetext{
* Nationales Naturhistorisches Museum der Bulgarischen Akademie der Wissenschaften, Boul. Ruski 1, Sofia, Bulgarien.
} 
Männchen hat halb so lange Elytren wie die männlichen Paratypen, was ich für eine Abnormität halte.

Die Typenserie ist in Alkohol konserviert; die inneren Genitalien zweier Männchen aus der Höhle Maara und zweier Männchen aus der Höhle Damlatas sind in $\mathrm{KOH}$ aufgehellt und auch in Alkohol konserviert. Alle Typen sind in den Sammlungen des Nationalen Naturhistorischen Museums der Bulgarischen Akademie der Wissenschaften, Sofia, aufbewahrt.

\section{Beschreibung der Männchen}

Körper gelblich bis gelbocker, alle Femora, die Ventralseite des Thorax und die Pleura heller, Elytren und die Hinterränder der Terga dunkler, ohne Flecke. Körper fein weiss dicht behaart, besonders auf der Unterseite, und spärlich mit dunklen Haaren und Borsten bedeckt.

Kopf schmäler als Pronotum. Erstes und zweites Glied der Maxillarpalpen kurz, drittes und viertes etwa gleichlang und fünftes am längsten. Scapus dreimal breiter als die übrigen Antennenglieder. Antennen 3,6 - 3,7 mal körperlang. Scapus in seiner grössten Breite fast zweimal bis genau zweimal so breit als Fastigium frontis zwischen den Fühlerbasen. Fastigium frontis mit einer Reihe von wenigen schwarzen Borsten beiderseits begrenzt.

Pronotum fast doppelt so breit wie lang, einfarbig, mit einer sehr dünnen hellen Mittellinie und abgerundeten Ecken, vor der Mitte am breitesten, am fast geraden Vorderrand und am leicht konkaven Hinterrand kräftig schwarz beborstet.

Elytren (Abb.1) lederig, ohne Adern, sehr fein behaart. Discus gelbbraun, von einem etwas dunkleren Band umrandet, das zur Basis des Elytrons heller wird. Ausserhalb dieses Bandes die hintere Hälfte des inneren Randes sowie der hintere und äussere Rand leicht hell gesäumt. Elytren konvex, gleichmässig rund, die Länge des ausserhalb des Pronotums sichtbaren Teils übertrifft um 0,2 mm ihre Maximalbreite und um 0,1 mm die Länge des Pronotums. Der Hinterrand der Elytren reicht ungefähr bis zum Hinterrand des Metanotums. Die Unterseite der Elytren mit Vertiefungen in Form von runden oder ovalen Grübchen oder Furchen, die auch auf der Oberseite durchschimmern.

Beine gelblich, alle Femora mit borstenförmigen Härchen. Vordertibia mit zwei Apikalsporen, die untere sind. Mitteltibia mit drei Apikalsporen, von denen eine obere innere und die beiden übrigen untere sind. Vorder- und Mitteltarsus kürzer als die entsprechenden Tibien. Vorder- und Mittelmetatarsus länger als die übrigen zwei Tarsenglieder zusammen. Hintertibia im Apikalteil dorsal aussen mit vier Dornen, von ihnen der vierte ganz kurz, der dritte am längsten, der zweite etwas kürzer oder gleich dem dritten und der erste kürzer oder gleich dem zweiten. Dorsal innen sind die Dornen drei oder vier; der erste von der vieren am kürzesten oder überhaupt nicht vorhanden, der vierte länger als der erste und befindet sich am Ende der Tibia ganz in der Nähe der Apikalsporen, der zweite etwas länger oder gleich dem vierten und der dritte am längsten. Die äusseren Apikalsporen der Hintertibia drei, von denen der obere am kürzesten, der mittlere am längsten und doppelt so lang wie der untere ist. Die inneren Apikalsporen auch drei, während der untere am 

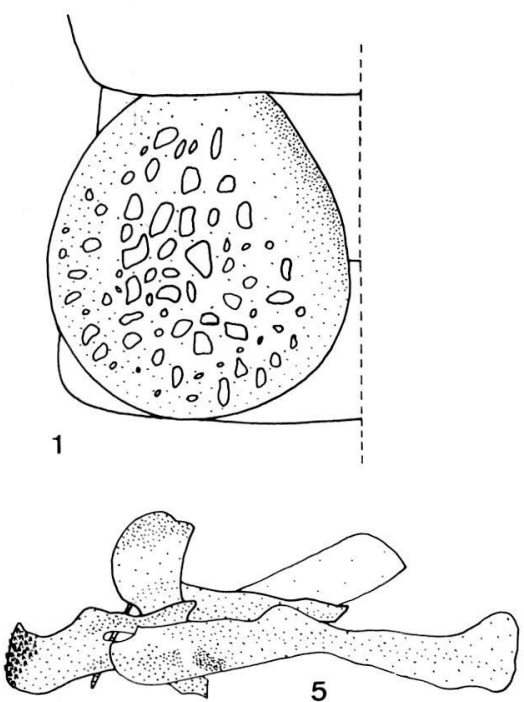

5
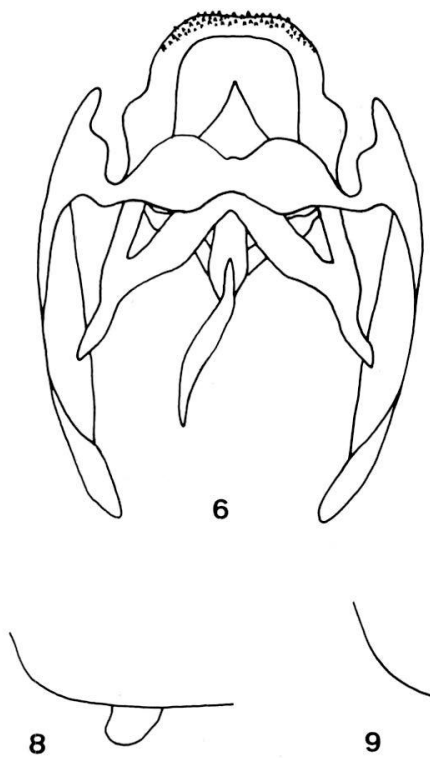
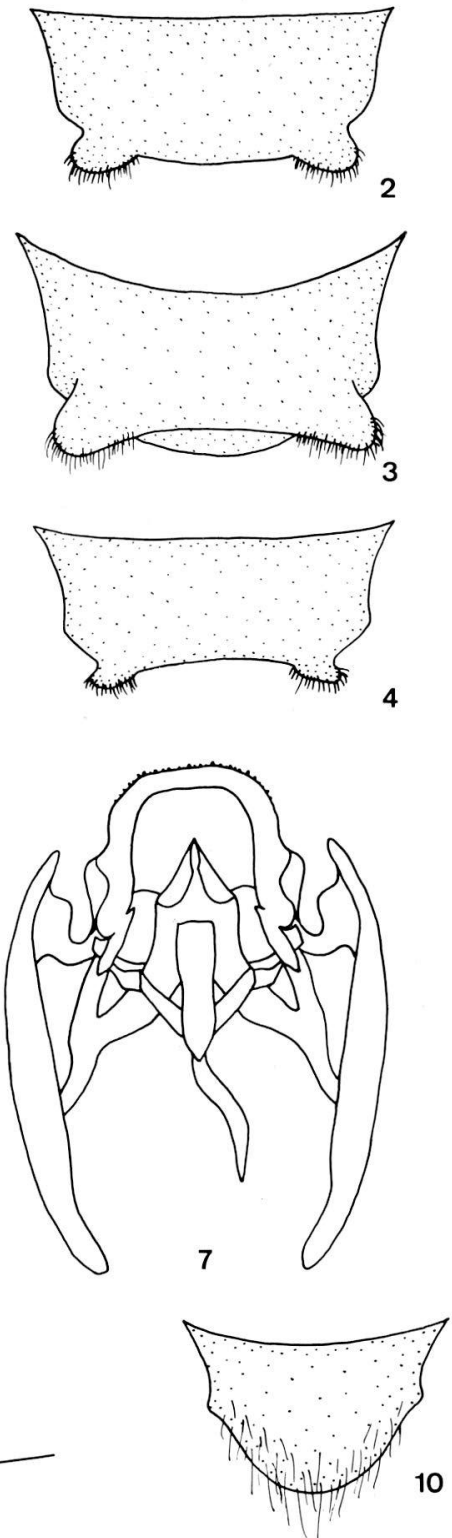

Abb. 1-7. Discoptila beroni n.sp., o: linkes Elytron (1); Epiproct (2-4); Genitalien, lateral (5), dorsal (6) urid ventral (7).

Abb. 8-10.Discoptila beroni n.sp., O: linke Ely tren (8 und 9); Epiproct (10).

Die Abbildungen 2, 3, 5-8 und 10 sind nach Material aus der Höhle Maara gezeichnet, die übrigen aus der Höhle Damlatas. 
kürzesten, der obere gleich oder etwas länger als der mittlere ist. Der untere innere Sporn der Hintertibia etwas länger als der untere äussere Sporn. Der äussere Sporn des hinteren Metatarsus so lang wie das zweite Tarsalglied und kürzer als der innere Sporn.

Epiproct (Abb. 2-4) quer, etwa doppelt oder mehr als doppelt so breit wie lang. Der Hinterrand des Epiprocts fast gerade, ohne Mittelvorsprung, mit behaarten nach aussen und hinten gerichteten abgerundeten vorspringenden Hinterecken. Die Breite der Hinterecken mehr als doppelt so gross wie der Abstand zwischen ihnen.

Die Genitalien sind aus Abb. 5-7 ersichtlich. Dorsal gesehen ist der Bogen des Epiphallus eng, nicht regelmässig gebogen, mit einem geraden Vorderrand; lateral gesehen ist er niedrig, mit einem konvexen Ventralrand. Die Dörnchen des Epiphallus fein. Der Apikalteil der ventral medianliegenden Struktur der Genitalien gerade, breitdreieckig. Das dorsale Querband (Abb. 6) büstenhalterförmig, mit deutlich ausgeprägten Konvexhemisphären; lateral gesehen ist es breit und hoch. Der asymmetrische Teil auf Abb. 6 und 7 sehr schwach sklerotisiert und bei anderen Exemplaren genau in der Medianebene liegend.

Körperlänge 11,6 - 13,1 mm, Länge der Antennen bis 48,5 mm, Pronotumlänge 1,7 - 2,0 mm, Länge der Elytren 1,8 - 2,2 mm, Länge der Hinterfemora 8,3 - 9,3 mm, Länge der Cerci $8,2-9,6 \mathrm{~mm}$.

\section{Beschreibung der Weibchen}

Körperfarbe, Kopf, Pronotum und Beine wie bei den Männchen.

Elytren (Abb. 8 und 9) winzig, als Elytrenreste stark reduziert, niemals von Pronotum verdeckt. Der sichtbare Teil hinter dem Pronotumrand breiter als lang. Die Form der Elytrenreste länglichoval, distal abgerundet.

Epiproct (Abb. 10) fast dreieckig, am Apex breit abgerundet. Cerci um $3 \mathrm{~mm}$ länger als Ovipositor. Ovipositor gerade, am Apex schnabelförmig zugespitzt, nicht punktiert. Seine Länge etwa zwei Drittel der Länge des Hinterfemurs. Der Ovipositor-Apex wie bei $D$. lindbergi Chop.

Körperlänge 10,8 - 14,2 mm, Länge der Antennen bis 52,5 mm, Pronotumlänge 2,0 - 2,4 mm, Länge der Elytren 0,1 - 0,3 mm, Länge der Hinterfemora 8,7 10,0 mm, Länge der Cerci 9,2 - 10,6 mm, Ovipositorlänge 5,3-6,9 mm.

\section{DISKUSSION}

Genitalmorphologisch unterscheiden sich die Männchen der Discoptila beroni n.sp. deutlich von den Arten mit bekannten Genitalien. In dieser Beziehung bestehen einige Aehnlichkeiten mit D. lindbergi Chop., D. brevis B.-Bien. und D. kinzelbachi Harz, bei $D$. beroni n.sp. jedoch besitzt das dorsale Querband (Abb. 6) symmetrische Wölbungen in Form von Konvexhemisphären. Habituell unterscheiden sich die Männchen der neuen Art von allen bekannten Discoptila-Arten wie folgendes: $D$. fragosoi (Bol.) und $D$. newmanae Harz besitzen fünf Dorne dorsal aussen auf der Hintertibia, hier sind diese Dorne vier; der Scapus ist bei D. kinzelbachi Harz kaum 
breiter, bei $D$. buresi Mar. mehr als doppelt so breit wie Fastigium frontis, bei der neuen Art fast doppelt; bei $D$. lindbergi Chop. sind die Hinterecken des Epiprocts nicht vorspringend, bei D. krueperi (Pant.) kaum vorspringend und bei D. brevis B.-Bien. nach hinten und nach innen gerichtet, hier nach hinten und nach aussen gerichtet. Die beiden Geschlechter der neuen Art haben keine Flecke auf Vertex, Pronotum und Hinterfemora, während D. zernyi (Wern.) solche hat. Im Vergleich zu allen übrigen Discoptila-Arten kennzeichnen sich die Männchen der D. beroni n.sp. durch die grössten Elytren, nicht nur absolut, sondern auch im Verhältnis zur Thoraxlänge. Bei den Weibchen bestehen ebenfalls deutliche Unterschiede zwischen der neuen Art und den Arten, deren Weibchen bekannt sind. Die Cerci sind kürzer als Ovipositor bei $D$. fragosoi und $D$. newmanae und länger bei der neuen Art; bei $D$. brevis ist der Ovipositor sehr kurz $(3,5 \mathrm{~mm})$, hier länger (über $5 \mathrm{~mm}$ ), bei $D$. lindoergi sind die Elytren unsichtbar, hier immer sichtbar hinter dem Rand des Pronotums.

Kritische Bemerkungen. Als erster teilt über den Fund einer Discoptila aus Kleinasien Ebner (1954) mit. Er stellt in dieser Gattung die von ihm im Jahre 1919 (Arch. Naturgesch., 85, A, 8: 155) beschriebenen Weibchen Gryllomorpha sp. ein, nach Weidrier (1964) aber gehören sie nicht zu Discoptila, da sie keine Elytrenreste haben. Folglich sind sie mit D. beroni n.sp. nicht identisch. Ebner (1954) schreibt weiter, dass vielleicht zwei Weibchen aus Libanon, von Pantel im Jahre 1890 (An. Soc.Esp.Hist.Nat., 19) erwähnt, und eventuell Gryllomorpha willemsei Uv. aus Aksehir (Uvarov, 1934) auch Discoptila sind. Die Gattungzugehörigkeit der ersten kann nach der Beschreibung nicht geklärt werden (Weidner, 1964) und das Männchen von $G r$. willemsei ist flügellos und nicht artengleich mit $D$. beroni n. sp. Die ersten Angaben über die hier beschriebene neue Art teilt Weidner (1964) nach Material aus der Höhle Damlatas unter den Namen D. fragosoi (Bol.) mit. Die Elytren der Männchen von D. fragosoi (aus Europa) sind aber oval, bis 1,5 mm lang, die Länge des Ovipositors bei den Weibchen beträgt über $7 / 8$ der Länge des Hinterfemurs, während bei meinem Material aus derselben Höhle die Elytren der Männchen rund, über 1,8 mm lang sind, und die Länge des Ovipositors $2 / 3$ der Länge des Hinterfemurs beträgt. Weitere Unterscheidungsmerkmale sind in der Diskussion angegeben. Auch Weidner (in litt.) meint, dass "dieses ist wahrscheinlich eine andere oder neue Art". Harz (1969) ordnet Exemplare aus demselben Fundort unter Frage zu D. brevis B.-Bien. ein. Er schreibt: “Aucn ơ aus Höhlen von Alanua und Damlatos (Türkei) scheinen hierher zu gehören". Von neuem wird die Höhle Damlatas bei Alanya (nicht "die Höhlen von Alanua und Damlatos" wie bei Harz, 1969) erwähnt. Harz hat aber nur männliche Exemplare besessen und hat sie mit einem Männchen von $D$. brevis aus Feodosia (Krim) verglichen, für das er die Möglichkeit nicht ausschliesst, einer anderen Art zu gehören (die Männchen von D. brevis waren zur Zeit nicht bekannt). Das Vorhandensein von Individuen beider Geschlechter in meinem Material gibt die Möglichkeit diese Einordnung zu widerlegen. Der Ovipositor des weiblichen Holotypus von D. brevis nach Bey-Bienko (1964) ist kürzer als die Hälfte des Hinterfemurs und nicht länger als $3,5 \mathrm{~mm}$, während bei $D$. beroni n.sp. er mehr als $5 \mathrm{~mm}$ lang ist und beträchtlich länger als die Hälfte des Hinterfemurs. 
Derivatio nominis. Die neue Art ist einem ihrer Sammler, meinem Freund Herrn Petar Beron aus dem Zoologischen Institut der Bulgarischen Akademie der Wissenschaften in Sofia, der sich rege mit der Höhlenfauna Bulgariens befasst, gewidmet.

Biotopangaben. Eine Beschreibung der Höhle Damlatas, ihre Lage und die Verhältnisse in ihr sowie die begleitende Fauna als Nahrungsquelle der Höhlengrillen gibt Weidner (1964) an. Seine Beschreibung stimmt mit der Beschreibung der Sammler vollkommen überein. Die letzten behaupten, dass der Boden der Höhle Maara mit Sand bedeckt ist und dass die Grillen in beiden Höhlen massenhaft zu finden waren.

Auf Grund der Oekologie und ihres Habitus kann D. beroni n.sp. für eine troglophile Art angenommen werden.

\section{ZUSAMMENFASSUNG}

Eine neue troglophile Höhlengrille, Discoptila beroni n.sp. wird beschrieben und abgebildet. Sie kommt in den Höhlen Maara bei Mersin und Damlatas bei Alanya in Südanatolien massenhaft vor. Es werden die Merkmale angegeben, nach denen die neue Art sich sowohl nach den Männchen als auch nach den Weibchen von den übrigen acht Discoptila-Arten, alle aus der Mediterraneis, unterscheidet. D. beroni n.sp. ist schon in der Literatur irrtümlicherweise als $D$. fragosoi (Bol.) und als $D$. brevis B.-Bien. aus der Höhle Damlatas mitgeteilt worden.

\section{LITERATUR}

Bey-Bienko, G., 1964. Ordnung Orthoptera (Saltatoria), Geradflügler. (Russisch). In: Bey-Bienko, G. (Herausg.). Opredelitel nassekomych Evropeiskoi tschasti SSSR, 1: 205-284, Leningrad.

Ebner, R., 1954. Orthopteroidea. In: Beier, M. Zoologische Studien in West-Griechenland. III. Teil. Sitzungsber. Oesterr.Akad. Wiss., Mathem.-naturw. Kl., Abt.I, 163 (8): 549-558, 6 Abb.

Harz, K., 1969. Die Orthopteren Europas, Volumen I. Dr. W. Junk N.V., Series entomologica, 5: 749 S., 2360 Abb., The Hague.

Uvarov, B., 1934. Studies in the Orthoptera of Turkey, Iraq and Syria. Eos, 10: 21-119.

Weidner, H., 1964. Discoptila fragosoi (I. Bolivar, 1885) aus einer Höhle Südanatoliens (Orthoptera: Gryllidae). Mitt. Deutsch. Ent. Ges., 23 (1): 17-20. 